Research Article

\title{
Preparation of Anodic Aluminum Oxide Masks with Size-Controlled Pores for 2D Plasmonic Nanodot Arrays
}

\author{
Mi Jung $\mathbb{D},{ }^{1}$ Ji-Hoon Kim, ${ }^{2}$ and Young-Wan Choi $\mathbb{D}^{2}$ \\ ${ }^{1}$ Institute of Innovative Functional Imaging, Chung-Ang University, Seoul 06974, Republic of Korea \\ ${ }^{2}$ School of Electrical and Electronics Engineering, Chung-Ang University, Seoul 06974, Republic of Korea \\ Correspondence should be addressed to Young-Wan Choi; ychoi@cau.ac.kr
}

Received 29 May 2018; Accepted 1 August 2018; Published 26 August 2018

Academic Editor: Nathan C. Lindquist

Copyright (C) $2018 \mathrm{Mi}$ Jung et al. This is an open access article distributed under the Creative Commons Attribution License, which permits unrestricted use, distribution, and reproduction in any medium, provided the original work is properly cited.

\begin{abstract}
Anodic aluminum oxide (AAO) layers with nano-sized pores have been used as shadow masks for the fabrication of two-dimensional (2D) metal nanodot arrays (NDAs). However, the localized surface plasmon resonance (LSPR) of size-controlled NDAs fabricated using AAO masks has not been much studied. In this study, we report on the improved preparation method and utilization of an ultrathin AAO mask for the fabrication of $2 \mathrm{D}$ plasmonic metal NDAs. The greatest challenge in preparing an AAO mask is to control the pore diameter and to make it reproducible. AAO masks with size-controlled pores were reproducibly prepared using a two-step chemical wet etching method. Ag NDAs with different dot diameters $(42,60$, and $80 \mathrm{~nm})$ and $\mathrm{Ag}, \mathrm{Cu}$, and $\mathrm{Au}$ NDAs with dot a diameter of $80 \pm 5 \mathrm{~nm}$ were fabricated on indium tin oxide glass substrates using AAO masks. The wavelengths corresponding to LSPR of 2D metal NDAs were investigated using ultraviolet-visible spectroscopy. Our results show that AAO masks with tunable pores can be used as shadow masks for the fabrication of $2 \mathrm{D}$ plasmonic NDAs.
\end{abstract}

\section{Introduction}

Fabrication techniques for two-dimensional (2D) plasmonic nanodot arrays (NDAs) have attracted significant attention in versatile plasmonic applications for the enhancement of the performance of optoelectronic devices and for the highly sensitive detection of biological and chemical sensors [1-3]. The localized surface plasmon resonance (LSPR) of metal nanoparticles is sensitive to parameters such as shape, size, composition, and the surrounding environment [4-6]. Along with the development of nanostructure manufacturing technology, research on various plasmonic applications based on the LSPR of metal nanostructures has been proceeding rapidly [7-12]. Plasmonic nanosensors have been utilized to detect chemical and biomolecular binding on metal surfaces by measuring extremely small wavelength shifts in LSPR owing to a change in the refractive index [7-9]. Plasmonic properties of metal nanoparticles have been investigated to improve the performance of optoelectronic devices such as organic light-emitting diodes (OLEDs), solar cells, and light-emitting diodes [10-12].
In addition, the LSPR features depending on the shape and size of metal nanostructures have a significant influence on the characterizations of optoelectronic devices such as the change of emission color in OLEDs, the conversion efficiency of solar energy, and the activity of catalyst [13-15]. Specifically, metal nanoparticle arrays exhibit much higher electromagnetic field enhancements than those of singleinsulated nanoparticles and are easy to reproducibly produce in the desired region $[16,17]$. Thus, manufacturing techniques that can reproduce a metal nanoparticle array on a substrate are important for optimizing the performance in plasmonic applications.

2D nanoparticle arrays such as Au disk, Ag disk, and ellipsoidal Au nanoparticles with different lattice constants have been achieved using electron beam lithography $[17,18]$. This technique facilitates the control of the geometric pattern of nanoparticles, but it has some drawbacks such as high cost and large-area patterning. Owing to these limitations, 2D metal nanoparticle arrays have been fabricated by nonlithographic techniques using self-organized nanostructured materials as a template $[4,8-11,19-27]$. Among the 

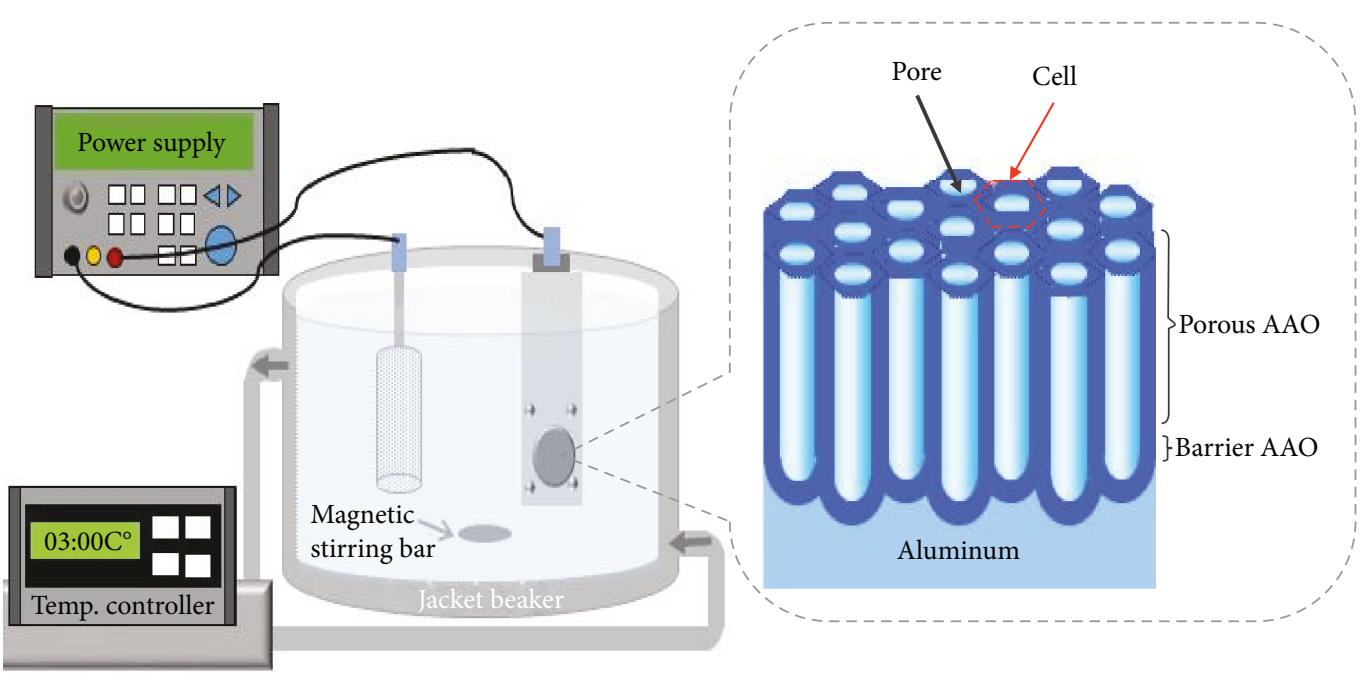

(a)

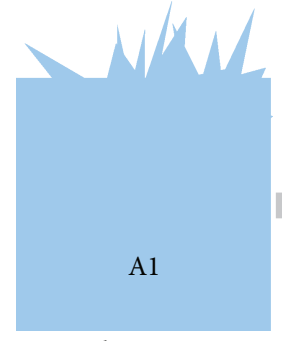

Aluminum

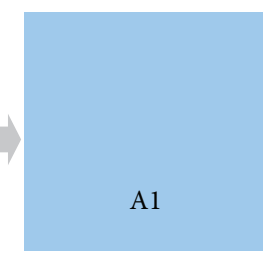

Electro-polishing

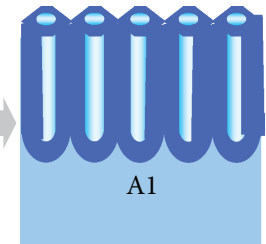

First anodization

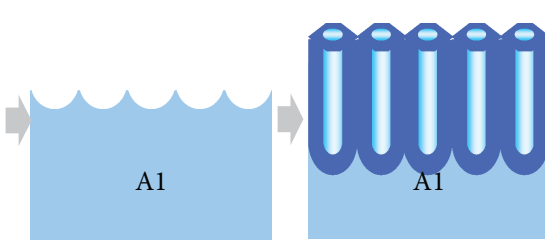

Etching
Second anodization

(b)

FIGURE 1: Schematic illustrations depicting the structure and the fabrication process for a well-ordered AAO layer: (a) the equipment configuration for the anodization method used in this work and (b) each experimental stage of the two-step anodization process.

materials, a self-organized anodic aluminum oxide (AAO) layer with uniform through holes of nanometer dimensions has been widely used as a shadow mask to fabricate 2D NDAs of various materials [20-27]. However, the plasmonic properties of metal NDAs produced using AAO masks have not been reported widely. Several studies have reported the LSPR of Ag or Au NDAs with height variations by varying the amount of metal deposited using AAO masks with similar pore diameters [24-26]. However, the LSPR of metal NDAs with different diameters of similar height has not yet reported due to the difficulty of controlling the pore diameter of the AAO mask. The most challenges in preparing the AAO mask are to completely remove the barrier layer at the bottom and control the pore diameter. In order to obtain an ultrathin AAO mask, the removal of the barrier layer and pore widening were performed by chemical etching in $5 \% \mathrm{H}_{3} \mathrm{PO}_{4}$ or by $\mathrm{Ar}^{+}$milling [24-30]. The $\mathrm{Ar}^{+}$ion milling method for the barrier layer removal is expensive and time-intensive [28]. Removing the barrier layer through long-term chemical etching is difficult to control the size of the pores because the AAO mask tends to break or crack [29]. In this study, we demonstrate an improved preparation method of AAO masks with adjustable pore diameters for the fabrication of size-controlled 2D NDAs. AAO masks were prepared by a two-step chemical wet etching method as reported previously [24]. The pore diameters of AAO masks were adjusted by controlling the dipping time in the second chemical wet etching process. $\mathrm{Ag}$ NDAs with different dot diameters and $\mathrm{Ag}, \mathrm{Cu}$, and $\mathrm{Au}$ NDAs with similar dot diameters were fabricated on indium tin oxide (ITO) substrates using AAO masks with sizecontrolled pores. The chemical structures of metal NDAs were examined by X-ray photoelectron spectroscopy (XPS). The plasmonic properties of metal NDAs were investigated using ultraviolet-visible spectroscopy.

\section{Experiments}

2.1. Preparation of $A A O$ Mask. The surface of the aluminum foil was pretreated using electrolytic polishing at $20 \mathrm{~V}$ for $60 \mathrm{~s}$ in a solution of perchloric acid and ethanol $\left(\mathrm{HClO}_{4}: \mathrm{C}_{2} \mathrm{H}_{6} \mathrm{O}=1: 5\right.$ (in volume ratio)). The aluminum foil inserted in a sample holder made of Teflon was connected to the anode, and the platinum electrode was connected to the cathode. Figure 1(a) shows the schematic diagrams of the equipment configuration for the anodization method and the structure of the AAO layer formed on the aluminum foil by the anodization method. Figure 1(b) shows the schematic diagrams of the two-step anodization process. The first anodization was performed in a $0.3 \mathrm{M}$ oxalic acid 
$\left(\mathrm{H}_{2} \mathrm{C}_{2} \mathrm{O}_{4}\right)$ solution for $6 \mathrm{~h}$ by applying a constant dc voltage of $40 \mathrm{~V}$. A magnetic stir bar was rotated at a constant speed at the bottom of the jacket beaker containing the solution to remove the heat generated during the anodization process. For low growth rates of the AAO layer, the temperature of the solution was maintained at $3^{\circ} \mathrm{C}$ using a refrigerated water circulator. The AAO layer formed during the first anodization process was dissolved in a mixed solution of $0.4 \mathrm{M}$ phosphoric acid $\left(\mathrm{H}_{3} \mathrm{PO}_{4}\right)$ and $0.2 \mathrm{M}$ chromic acid $\left(\mathrm{H}_{2} \mathrm{CrO}_{4}\right)$ at $65^{\circ} \mathrm{C}$ for approximately $6 \mathrm{~h}$. The second anodization was performed for $240 \mathrm{~s}$ under the same conditions as those of the first anodization. The AAO layer was etched slightly via immersion for $600 \mathrm{~s}$ in $5 \mathrm{wt} \%$ phosphoric acid at $30^{\circ} \mathrm{C}$ following the second anodization. The surface of the AAO layer was subsequently coated with a solution containing a mixture of nitrocellulose and polyester resin in butyl acetate, ethyl acetate, and isopropyl alcohol. The remaining aluminum substrate was removed using a saturated mercuric chloride $\left(\mathrm{HgCl}_{2}\right)$ solution for approximately $4 \mathrm{~h}$. The pore diameters of AAO masks were adjusted by controlling the dipping time in the second chemical wet etching process. Finally, the coating layer on the AAO surface was dissolved in acetone. Subsequently, the AAO mask was filtered using a filter paper.

2.2. Fabrication of Plasmonic NDAs. The AAO mask was placed on an ITO substrate, which was subsequently placed on a sample holder in the evaporator chamber. Metal was deposited on the surface of the ITO substrate covered with the AAO mask by using an electron beam evaporator (ULVAC EBX-1000). Under a vacuum of $\sim 10^{-4}$ Torr, metals such as $\mathrm{Ag}, \mathrm{Cu}$, and $\mathrm{Au}$ were deposited at a deposition rate of $\sim 0.1 \mathrm{~nm} / \mathrm{s}$ for $500 \mathrm{~s}$. Under these deposition conditions, the thickness of the metal film deposited on the ITO substrate without an AAO mask was approximately $50 \mathrm{~nm}$. After the deposition of each metal, the mask on the ITO substrate was selectively dissolved in $1 \mathrm{M}$ sodium hydroxide $(\mathrm{NaOH})$ for several minutes and rinsed several times with distilled water. Thus, $\mathrm{Ag}, \mathrm{Cu}$, and $\mathrm{Au}$ NDAs were formed on the ITO substrate.

2.3. Characterizations. The morphologies of the AAO mask and metal NDAs were observed using a field-emission scanning electron microscope (FE-SEM; Hitachi S-4700) and atomic force microscope (AFM; Park Systems XE-100). The chemical compositions and electronic structures of the metal NDAs were confirmed using XPS (PHI 5000 Versa Probe, ULVAC-PHI Inc.). The XPS measurements were performed using microfocused $(100 \mu \mathrm{m} \times 100 \mu \mathrm{m}, 25 \mathrm{~W}$, $15 \mathrm{kV}$ ) monochromatic $\mathrm{Al} \mathrm{K} \alpha$ radiation with an energy of $1486.6 \mathrm{eV}$. The instrument was calibrated to provide a $\mathrm{C} 1 \mathrm{~s}$ binding energy of $284.6 \mathrm{eV}$. The pressure inside the analyzer was maintained at $5 \times 10^{-10}$ Torr. The plasmonic properties of the metal NDAs were measured using a UV-Vis spectrometer (Perkin Elmer Lambda 18) in a wavelength range of $270-900 \mathrm{~nm}$. The sample was placed behind a collimator lens and on a position-controlled stage, and the extinction measurement was recorded using unpolarized light with a probe beam size of approximately $4 \mathrm{~mm}^{2}$.

\section{Results and Discussion}

3.1. Preparation of AAO Mask. The AAO layer was prepared from an aluminum foil by the two-step anodization process reported by Masuda and Satoh [22]. After the second anodization, the AAO layer was immersed once in the phosphoric acid solution, and then the pore diameter of the AAO mask was controlled by varying the immersion time in the second etching process. The AAO mask with size-controlled pores was prepared by a two-step wet etching process, which was designed with the hint in the two-step anodization method. Figure 2 shows schematic illustrations and FE-SEM images of the pore opening process of the AAO layer using the two-step wet etching method. Figure 2(a) shows the FE-SEM image of the cross-sectional view of the AAO layer formed via the second anodization for $4 \mathrm{~min}$. The structure of the AAO layer was composed of a porous layer and barrier layer on the aluminum substrate. The porous layer consisted of densely packed cylindrical pores perpendicular to the barrier layer. The thickness of the AAO layer was approximately $250 \mathrm{~nm}$. The barrier layer at the bottom of the AAO layer had a uniform thickness of $46 \pm 3 \mathrm{~nm}$ for each cell. The close-packed hexagonal cell size of the AAO layer is approximately $105 \pm 5 \mathrm{~nm}$. After the first wet etching conducted in $5 \mathrm{wt} \% \mathrm{H}_{3} \mathrm{PO}_{4}$ solution for $600 \mathrm{~s}$, the barrier layer thickness at the bottom of the AAO layer is approximately $26 \mathrm{~nm}$ as shown in Figure 2(b). In a comparison of Figures 2(a) and 2(b), Figure 2(b) clearly shows a thinner barrier thickness. Figure 2(c) shows the FE-SEM image of the bottom of the alumina barrier layer after the remaining aluminum substrate was dissolved in a saturated $\mathrm{HgCl}_{2}$ solution after the first wet etching for $600 \mathrm{~s}$. The most important step in preparing an AAO mask is to completely remove the barrier AAO layer and to open the pores. After the first wet etching, the surface of the AAO layer was coated with the coating layer, and the remaining aluminum substrate was removed using a saturated $\mathrm{HgCl}_{2}$ solution. In the second wet etching process, the AAO layer was immersed again in $5 \mathrm{wt} \% \mathrm{H}_{3} \mathrm{PO}_{4}$ solution for $690 \mathrm{~s}$. Figure 2(d) shows the FE-SEM image of the AAO mask after the removal of the protecting layer in acetone. As shown in Figure 2(d), it was confirmed that the barrier AAO layer was completely dissolved. The pores were widened uniformly, and the average diameter of the pores was approximately $60 \mathrm{~nm}( \pm 5 \mathrm{~nm})$.

3.2. Metal NDAs Using AAO Masks. Ultrathin, large-area AAO masks could be prepared using a two-step wet etching process. Au was deposited on an ITO substrate covered with an AAO mask for $500 \mathrm{~s}$ at a deposition rate of $0.1 \mathrm{~nm} / \mathrm{s}$. Figure 3(a) shows the FE-SEM image of Au-deposited AAO mask and Au NDA revealed from which the AAO mask was partially stripped with a carbon tape. Figure 3(b) shows the FE-SEM image of Au NDA after removing the AAO mask with a carbon tape. Figure 3(c) shows the interface between the Au-deposited mask surface and the Au NDA after the AAO mask is partially removed. Figure 3(d) shows that $\mathrm{Au}$ clusters were deposited on the top surface of the AAO mask as a replica of the pore pattern of the mask. Under 


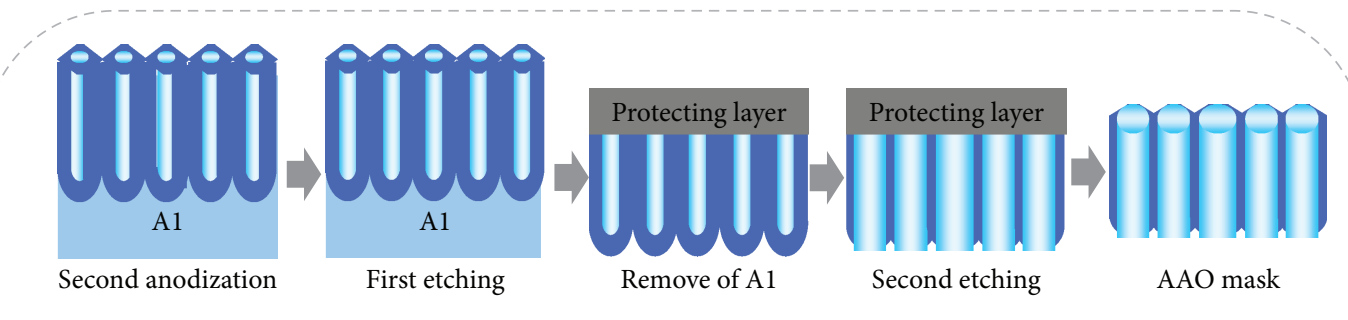

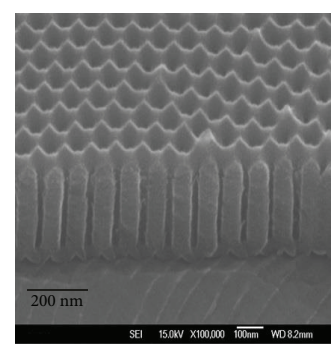

(a)

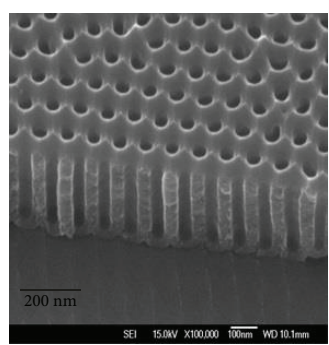

(b)

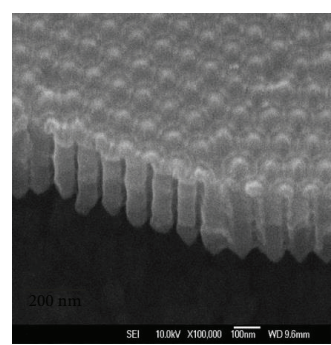

(c)

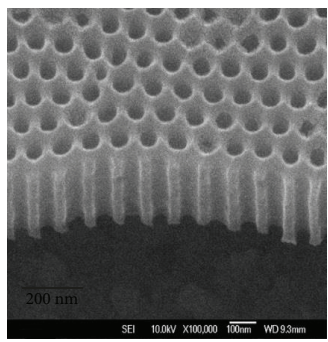

(d)

FIGURE 2: Schematic illustrations depicting the two-step wet etching process. FE-SEM images show the AAO layer prepared in the pore opening process using the method: (a) the second anodization for $4 \mathrm{~min}$, (b) the first chemical wet etching for $10 \mathrm{~min}$, (c) the bottom part of the AAO layer after dissolving the remaining aluminum substrate in a saturated $\mathrm{HgCl}_{2}$ solution, and (d) the AAO layer with through pores after the second wet etching for $11 \mathrm{~min}$.

the deposition rate of $0.1 \mathrm{~nm} / \mathrm{s}$, it can be observed that $\mathrm{Au}$ was uniformly deposited without blocking the nanopores of the AAO mask.

Figure 4(a) shows that the pore diameter of the AAO mask etched by the second dipping for $600 \mathrm{~s}$ was $43 \mathrm{~nm}$ $( \pm 5 \mathrm{~nm})$ after the first wet etching for $600 \mathrm{~s}$. Figures $4(\mathrm{~b})$ and 4(c) show the FE-SEM images of the AAO mask with different pore diameters depending on the second wet etching time, that is, $60 \mathrm{~nm}( \pm 5 \mathrm{~nm})$ for the dipping time of $690 \mathrm{~s}$ and $80 \mathrm{~nm}( \pm 5 \mathrm{~nm})$ for the dipping time of $780 \mathrm{~s}$. After the deposition of $\mathrm{Ag}$, the AAO masks were dissolved in $1 \mathrm{M}$ $\mathrm{NaOH}$ solution. The Ag NDAs with size-controlled diameters of $45 \pm 5 \mathrm{~nm}$ (Figure 4(d)), $60 \pm 5 \mathrm{~nm}$ (Figure 4(e)), and $80 \pm 5 \mathrm{~nm}$ (Figure 4(f)) were fabricated using the AAO masks with different pore diameters. After the first wet etching for $600 \mathrm{~s}$, the pore diameters of the AAO masks could be controlled by varying the dipping time.

Figures 5(a)-5(c) show the topology of Ag NDAs with different dot sizes investigated using AFM. The crosssectional line profile along the red line in the AFM topographic image of Ag NDA is shown in Figure 5(a), with an average height of $20 \pm 5 \mathrm{~nm}$ corresponding to the dots on the line. The mean diameter of Ag NDA formed on the ITO substrate was $45 \pm 5 \mathrm{~nm}$. Figure 6(b) shows the crosssectional line profile along the red line in the AFM topology image of the curve, with an average height of $22 \pm 5 \mathrm{~nm}$ corresponding to the vertices of two points on this line. The mean diameter of $\mathrm{Ag} \mathrm{NDA}$ was $60 \pm 5 \mathrm{~nm}$. In the curved AFM topology image, the cross-sectional line profile along the red line is shown in Figure 5(c), with an average height of $18 \pm 4 \mathrm{~nm}$ corresponding to the two vertices of the point. The average diameter of Ag NDA was $80 \pm 5 \mathrm{~nm}$. Considering the variation in height at the nanolevel of the surface of the coated ITO substrate, it can be observed that the results were produced at approximately the same height. The dot heights of Ag NDA depend on the aspect ratio of the AAO mask and the amount of the deposited metal $[20,26]$. Thus, it can be observed that AAO masks were manufactured under similar conditions.

Figures 6(a)-6(c) show the FE-SEM images of $\mathrm{Ag}, \mathrm{Cu}$, and $\mathrm{Au}$ NDAs with similar diameters that were fabricated on ITO substrates using AAO masks with a pore diameter of $80 \pm 5 \mathrm{~nm}$. The average diameter of the metal NDAs was $80 \pm 5 \mathrm{~nm}$ as a replica of the AAO mask. The chemical composition and bonding states of $\mathrm{Ag}, \mathrm{Cu}$, and $\mathrm{Au}$ NDAs were studied using XPS. The binding energies were referenced to the C1s line, which was at $284.6 \mathrm{eV}$ from carbon. As shown in Figure 6(d), the XPS spectra obtained from Ag NDA were centered at 368.2 and $374.2 \mathrm{eV}$. These values are consistent with the $\mathrm{Ag} 3 \mathrm{~d}_{5 / 2}$ and $\mathrm{Ag} 3 \mathrm{~d}_{3 / 2}$ core-level binding energies for the $\mathrm{Ag}$ nanoparticles appearing at 368.1 and $374.1 \mathrm{eV}$ [31]. The separation value between the $\mathrm{Ag} 3 \mathrm{~d}_{5 / 2}$ and $\mathrm{Ag} 3 \mathrm{~d}_{3 / 2}$ transitions exhibits a spin orbital splitting of $6.0 \mathrm{eV}$, which is a characteristic of metallic silver [32]. Figure 6(e) shows the XPS spectra obtained from $\mathrm{Cu}$ NDA. The XPS spectra of $\mathrm{Cu} 2 \mathrm{p}_{3 / 2}$ at $932.4 \mathrm{eV}$ and $2 \mathrm{p}_{1 / 2}$ at $952.4 \mathrm{eV}$ confirm the presence of metallic copper [33, 34]. Otherwise, the two dominant peaks were $\mathrm{Cu} 2 \mathrm{p}_{1 / 2}$ at $953.9 \mathrm{eV}$ with an intense satellite at $962.5 \mathrm{eV}$ and $2 \mathrm{p}_{3 / 2}$ at $934.4 \mathrm{eV}$ with an intense satellite at $941.2 \mathrm{eV}$, which are the peaks corresponding to $\mathrm{CuO}$ and copper hydroxide $\left(\mathrm{Cu}(\mathrm{OH})_{2}\right)$. Copper hydroxide usually displays the $\mathrm{Cu}$ $2 \mathrm{p}_{3 / 2}$ spectrum in the range of 934 to $936 \mathrm{eV}[34,35]$. The values of the $\mathrm{Cu} 2 \mathrm{p}_{3 / 2}$ binding energies for $\mathrm{Cu}$ metals, $\mathrm{CuO}, \mathrm{Cu}(\mathrm{OH})_{2}$, and $\mathrm{Cu}_{2} \mathrm{O}$ obtained from previous literature are 932.67, 932.6, 933.7, and $934.9 \mathrm{eV}$, respectively [36]. 


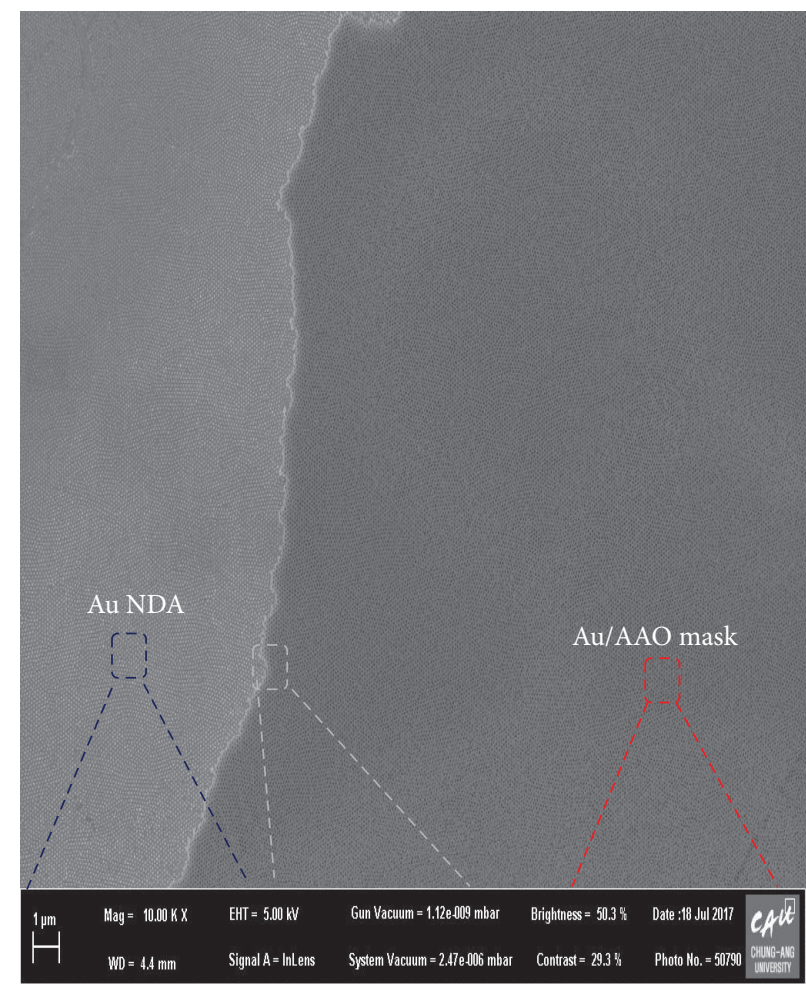

(a)

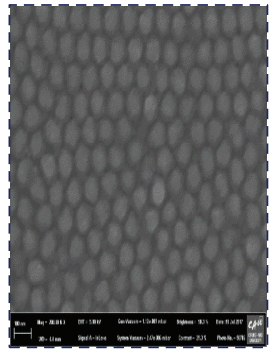

(b)

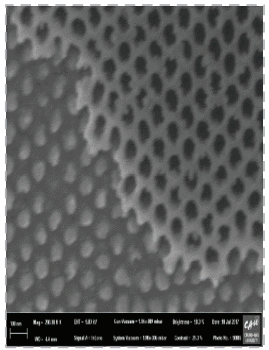

(c)

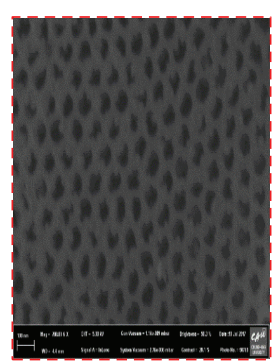

(d)

Figure 3: FE-SEM images of (a) Au-deposited AAO mask and Au NDA after a portion of the AAO mask was removed with a carbon tape, (b) Au NDA, (c) the boundary between the Au-deposited AAO mask and Au NDA, and (d) Au clusters deposited on top of the AAO mask.

These results indicate that copper hydroxide was formed on the surfaces of the $\mathrm{Cu}$ NDA during the process of dissolution of the AAO mask in aqua solution. As shown in Figure 6(f), the $\mathrm{Au} 4 \mathrm{f}_{7 / 2}$ and $\mathrm{Au} 4 \mathrm{f}_{5 / 2}$ core-level binding energies for $\mathrm{Au}$ NDA were observed at 84.1 and $87.9 \mathrm{eV}$. The binding energy of $\mathrm{Au} 4 \mathrm{f}_{7 / 2}$ for metallic gold was observed at $84.0 \mathrm{eV}$ [37]. The binding energies of $\mathrm{Au} 4 \mathrm{f}_{7 / 2}$ and $\mathrm{Au} 4 \mathrm{f}_{5 / 2}$ for the $\mathrm{Au}$ NDA were observed at 84.7 and $88.3 \mathrm{eV}$, respectively [16]. The slight difference in the XPS peak position may be due to the changes in binding energy because of the particle shape or local chemical composition on the sample surface [37]. Thus, the material components of plasmonic NDAs were confirmed using XPS measurement.

3.3. Plasmonic Properties of Metal NDAs. The extinction spectrum, which is the sum of the absorption and scattering spectra, of metal NDA was measured in transmission mode using UV-Vis spectroscopy. The optical extinction has a maximum at the plasmon resonant frequency, which corresponds to the LSPR peak of noble metal nanoparticles appearing in the visible wavelength range [6]. Figure 7(a) shows the LSPR spectra of Ag NDAs with different dot diameters in the period of $105 \mathrm{~nm}$. The LSPR spectrum of the Ag NDA with a diameter of $45 \pm 5 \mathrm{~nm}$ indicates the maximum peak at $498 \mathrm{~nm}$. The spectra of the Ag NDAs with diameters $60 \pm 5 \mathrm{~nm}$ and $80 \pm 5 \mathrm{~nm}$ indicate the maximum peaks at $516 \mathrm{~nm}$ and $533 \mathrm{~nm}$. As shown in Figure 7(a), the LSPR peak position is observed at different positions depending on the Ag dot size, shifting to the red region as the Ag nanodot diameter increases. The LSPR intensity of an Ag NDA with larger dots is higher than that with smaller dots. The LSPR peak broadening for Ag NDA on the substrate becomes larger as the diameter of the nanodots increased. The linewidth and the amplitude of the LSPR peaks depend on the size distribution and the distances between the particles $[38,39]$. Our results are similar to those of the previous 


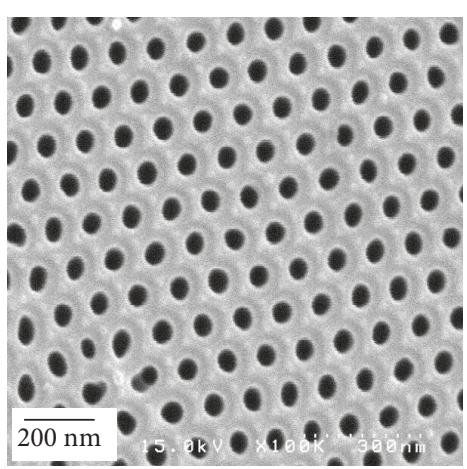

(a)

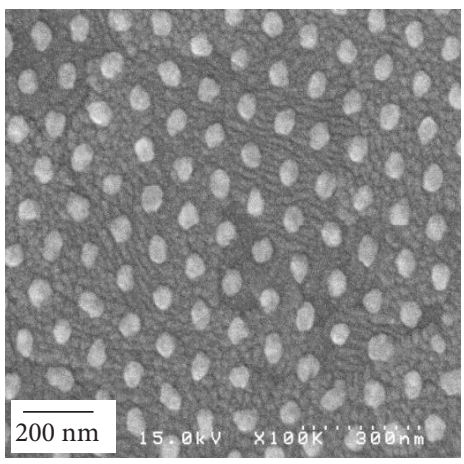

(d)

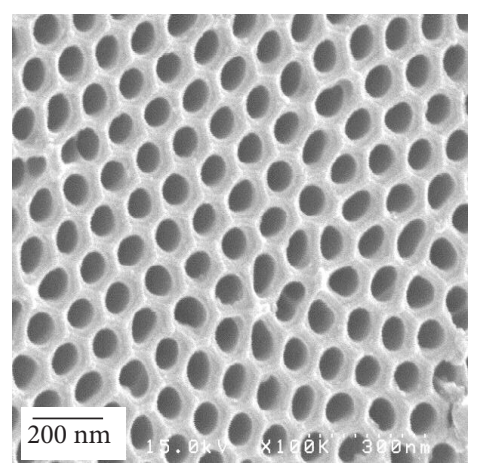

(b)

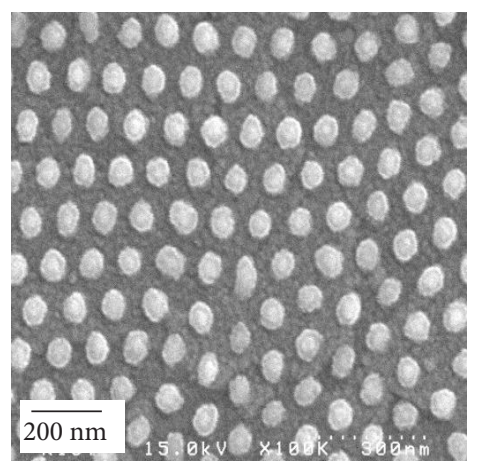

(e)

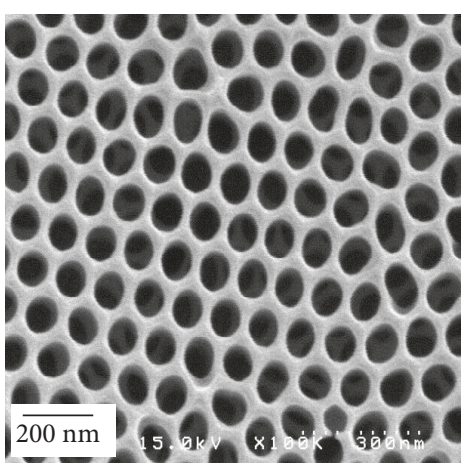

(c)

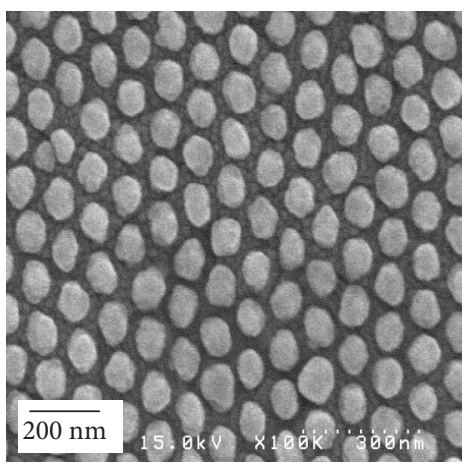

(f)

FIGURE 4: FE-SEM images of the AAO mask with different diameters: (a) $45 \mathrm{~nm}$, (b) $60 \mathrm{~nm}$, and (c) $80 \mathrm{~nm}$. FE-SEM images of Ag NDAs with different diameters-(d) $42 \mathrm{~nm}$, (e) $60 \mathrm{~nm}$, and (f) $80 \mathrm{~nm}$-formed on ITO substrates.

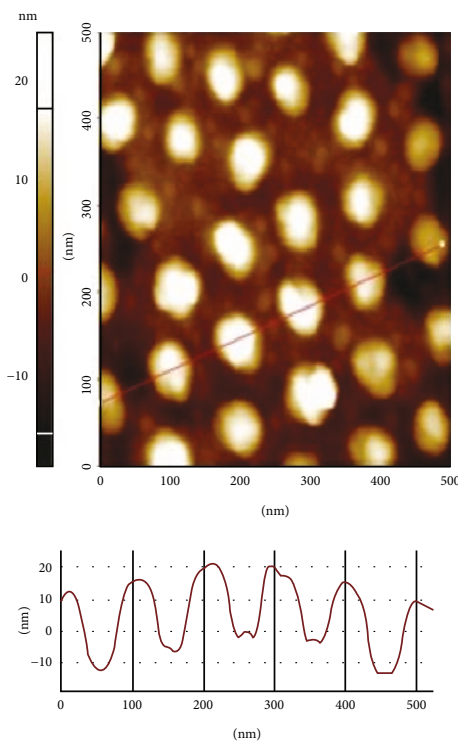

(a)
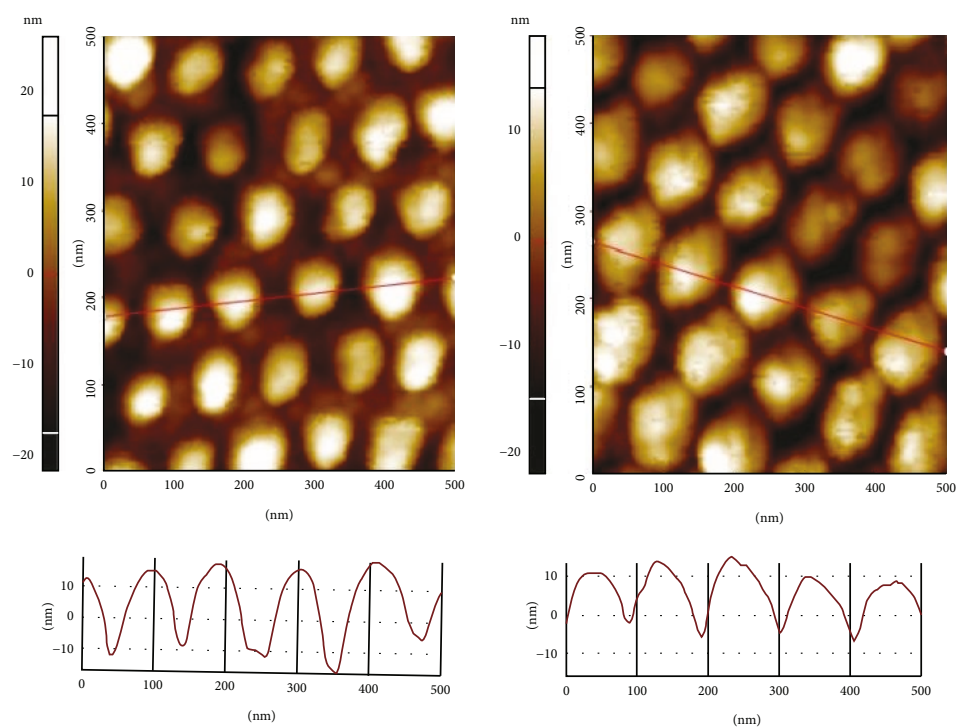

(b)

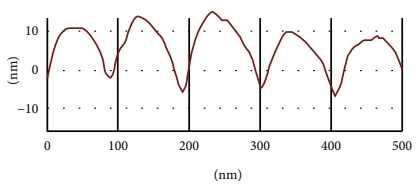

(c)

FIgURE 5: 2D AFM images of top views and cross-sectional line profiles in red lines of Ag NDAs with different diameters: (a) $45 \mathrm{~nm}$, (b) $60 \mathrm{~nm}$, and (c) $80 \mathrm{~nm}$.

studies in which the LSPR wavelength shifts to red and the peak broadens as the particle size increases [4, 18]. Figure 7(b) shows the LSPR spectra of $\mathrm{Ag}, \mathrm{Cu}$, and $\mathrm{Au}$ NDAs with similar dot diameters of $80 \pm 5 \mathrm{~nm}$. Although the sizes of metal NDAs were similar, the maximum peaks of LSPR were observed at different positions depending on the native element of the metal. The spectrum for the Ag NDA shows the maximum peak at $533 \mathrm{~nm}$. For the spectra 


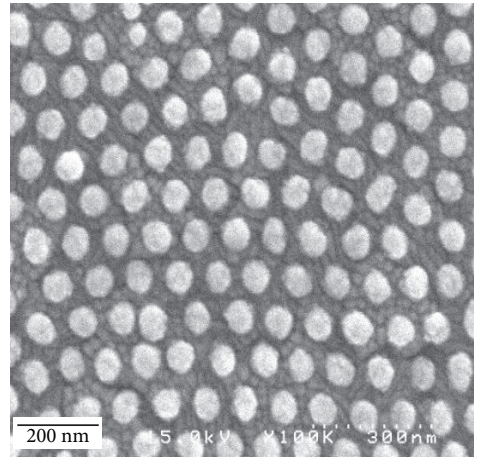

(a)

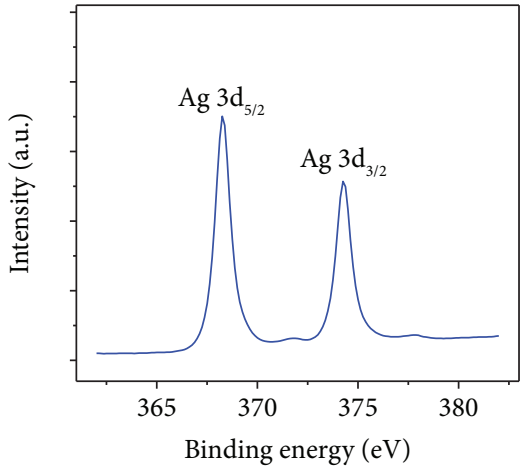

(d)

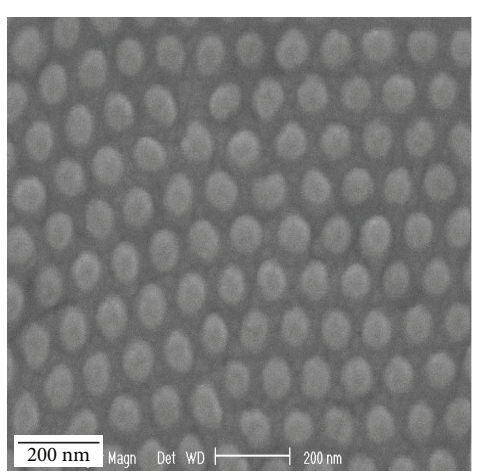

(b)

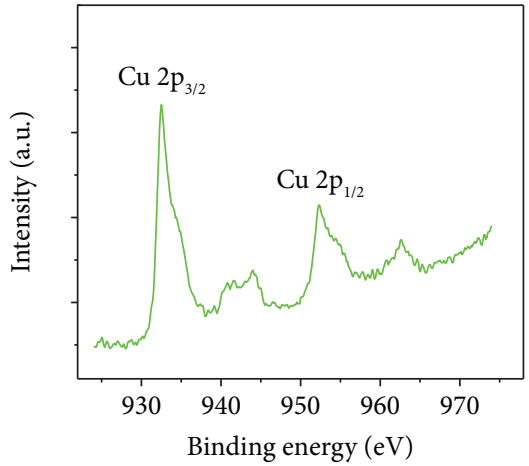

(e)

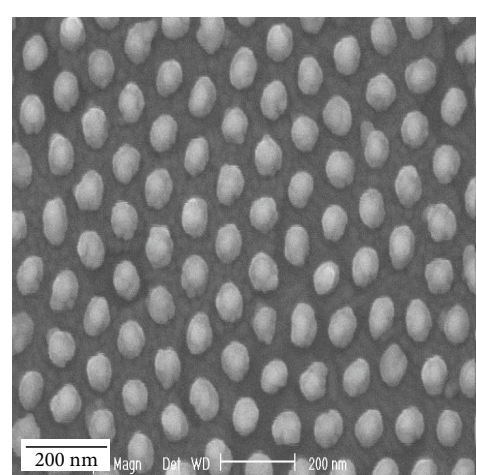

(c)

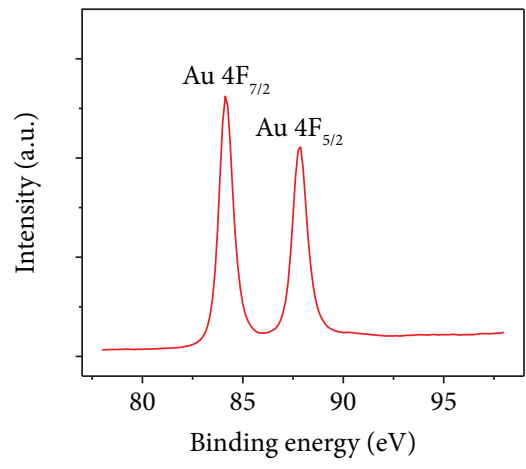

(f)

Figure 6: FE-SEM images of (a) Ag, (b) Cu, and (c) Au NDA with a diameter of $80 \mathrm{~nm}$. The XPS spectra obtained from (d) 3d levels of Ag NDA, (e) $2 p$ levels of Cu NDA, and (c) $4 \mathrm{f}$ levels of Au NDA.

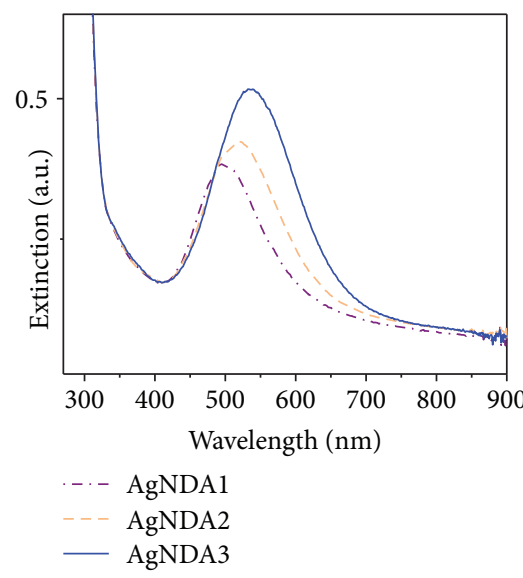

(a)

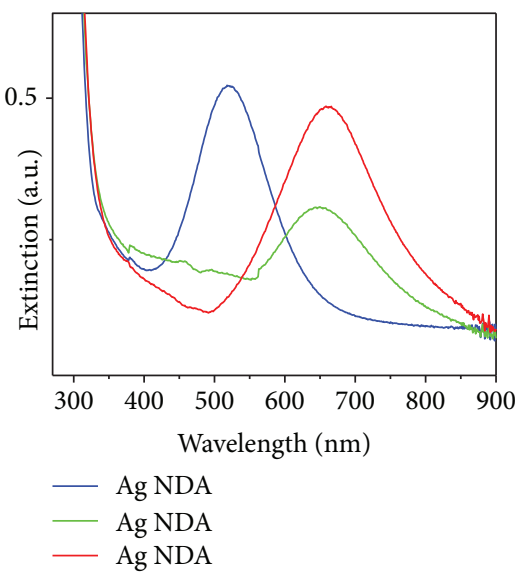

(b)

FIgURE 7: Extinction spectra of (a) Ag NDAs with different dot sizes (AgNDA1: $D=42 \mathrm{~nm}$, AgNDA2: $D=60 \mathrm{~nm}$, and AgNDA2: $D=80 \mathrm{~nm}$ ) and (b) $\mathrm{Ag}, \mathrm{Cu}$, and $\mathrm{Au}$ NDAs with a similar dot diameter $(D=\sim 80 \mathrm{~nm})$.

of the $\mathrm{Cu}$ and $\mathrm{Au}$ NDAs, the maximum peaks were shown at $648 \mathrm{~nm}$ and $673 \mathrm{~nm}$, respectively. The LSPR peak of the Ag NDAs was observed at a shorter wavelength than those of the $\mathrm{Cu}$ and $\mathrm{Au}$ NDAs. This result is consistent with the trends of peak positions of the LSPR from $\mathrm{Ag}$ and $\mathrm{Au}$ nanoparticles [40].

The LSPR of metal NDAs prepared using AAO masks has mainly focused on Ag or Au NDAs of different heights with similar pore diameters [24-26]. Au NDAs of different heights $(25,40,60$, and $80 \mathrm{~nm})$ with a similar dot diameter of $55 \mathrm{~nm}$ were fabricated on quartz plates, and the LSPR peaks are located at $700,650,620$, and $585 \mathrm{~nm}$ [24]. The positions of LSPR peaks show a blue shift upon increasing the height of Au NDAs. Ag NDAs of different heights $(75,45$, and $25 \mathrm{~nm})$ with a similar dot diameter of $65 \mathrm{~nm}( \pm 4 \mathrm{~nm})$ were fabricated on the glasses, and the LSPR 
peaks are observed at 433,452 , and $502 \mathrm{~nm}$. The peak positions of the Ag NDAs with $\sigma=0.88,1.56$, and 2.44 shift to higher wavelengths as the aspect ratio increases [25]. The calculated peak broadening is smaller than the experimental width of the LSPR peak for the Ag NDA on the glass substrates. This is due to the dispersion of geometrical dimensions of the nanodots in the actual samples $[25,38]$. The LSPR characteristics of metal NDAs are significantly affected by the substrate, metal deposition conditions, and the aspect ratio of the AAO mask $[6,20]$. In addition, the LSPR in conductive metal oxide nanoparticles and in vacancy-doped semiconductor quantum dots is strongly influenced by the free electron carrier density [41, 42]. Since LSPR wavelengths are affected by various factors such as the nanoparticle size, composition, and substrate, free electron carrier density, more experiments should be systematically conducted to explore the LSPR in many material systems. To do this, reproducible manufacturing techniques of AAO masks are important. In the conventional wet etching process, the immersion time required for the pore opening of the AAO layer was difficult to control well owing to a slight difference in the thickness of the AAO barrier layer [29]. The etching rate may vary depending on the diffusion rate of the acid and depending on the shape of the surface, and hence even though there is a very small difference in height, the shape of the pores is significantly affected by etching for a long time [30]. A conventional chemical wet etching method for a long time results in the pore opening of the alumina barrier layer, but the pore wall of the AAO layer is sometimes collapsed. By using a two-step wet etch process, it was possible to achieve the pore opening in a short time without breaking the pore wall of the AAO layer, and it was possible to stably and reproducibly prepare AAO masks with size-controlled pores.

\section{Conclusions}

We demonstrated reproducible fabrication and plasmonic properties of size-controlled plasmonic NDAs using AAO masks. Large-area nanoporous alumina masks were achieved by a two-step wet etching process. The pore diameter of the $\mathrm{AAO}$ mask can be reproducibly controlled in the range of the hexagonal cell size by varying the immersing time of the second chemical wet etching. The dot diameter of the metal nanodot array depends on the AAO mask used as the evaporation mask. The LSPR spectra of Ag NDAs with different sizes $(42 \mathrm{~nm}, 60 \mathrm{~nm}$, and $80 \mathrm{~nm})$ showed the maximum peak at $498 \mathrm{~nm}, 516 \mathrm{~nm}$, and $533 \mathrm{~nm}$ and shifted to the red region as the dot diameter increased. The LSPR spectra for the $\mathrm{Ag}, \mathrm{Cu}$, and $\mathrm{Au}$ NDAs with a similar diameter of approximately $80 \pm 5 \mathrm{~nm}$ indicated the maximum peaks at $533 \mathrm{~nm}, 648 \mathrm{~nm}$, and $673 \mathrm{~nm}$. These AAO masks could be utilized as a versatile mask to manufacture various $2 \mathrm{D}$ plasmonic NDAs.

\section{Data Availability}

All the data supporting the results are shown in the paper and are available from the corresponding author upon request.

\section{Conflicts of Interest}

There are no conflicts to declare.

\section{Acknowledgments}

This research was supported by the Basic Science Research Program through the National Research Foundation (NRF) of South Korea funded by the Ministry of Education (NRF-2015R1D 1A 1A01058692). This research was supported by the Chung-Ang University Research Scholarship grants in 2016. The authors would like to thank Dr. Seok Lee and Deok Ha Woo of the Korea Institute of Science and Technology for their helpful discussions.

\section{References}

[1] M. Jung, D. Mo Yoon, M. Kim et al., "Enhancement of hole injection and electroluminescence by ordered Ag nanodot array on indium tin oxide anode in organic light emitting diode," Applied Physics Letters, vol. 105, no. 1, article 013306, 2014.

[2] O. Vazquez-Mena, T. Sannomiya, L. G. Villanueva, J. Voros, and J. Brugger, "Metallic nanodot arrays by stencil lithography for plasmonic biosensing applications," ACS Nano, vol. 5, no. 2, pp. 844-853, 2011.

[3] Y. Lin, Y. Zou, Y. Mo, J. Guo, and R. G. Lindquist, "E-beam patterned gold nanodot arrays on optical fiber tips for localized surface plasmon resonance biochemical sensing," Sensors, vol. 10, no. 10, pp. 9397-9406, 2010.

[4] T. R. Jensen, M. D. Malinsky, C. L. Haynes, and R. P. van Duyne, "Nanosphere lithography: tunable localized surface plasmon resonance spectra of silver nanoparticles," The Journal of Physical Chemistry B, vol. 104, no. 45, pp. 1054910556, 2000.

[5] K. L. Kelly, E. Coronado, L. L. Zhao, and G. C. Schatz, "The optical properties of metal nanoparticles: the influence of size, shape, and dielectric environment," Physical Chemistry B, vol. 107, no. 3, pp. 668-677, 2003.

[6] M. Duval Malinsky, K. L. Kelly, G. C. Schatz, and R. P. van Duyne, "Nanosphere lithography: effect of substrate on the localized surface plasmon resonance spectrum of silver nanoparticles," The Journal of Physical Chemistry B, vol. 105, no. 12, pp. 2343-2350, 2001.

[7] A. J. Haes and R. P. Van Duyne, "A nanoscale optical biosensor: sensitivity and selectivity of an approach based on the localized surface plasmon resonance spectroscopy of triangular silver nanoparticles," Journal of the American Chemical Society, vol. 124, no. 35, pp. 10596-10604, 2002.

[8] K. A. Willets and R. P. Van Duyne, "Localized surface plasmon resonance spectroscopy and sensing," Annual Review of Physical Chemistry, vol. 58, no. 1, pp. 267-297, 2007.

[9] J. N. Anker, W. P. Hall, O. Lyandres, N. C. Shah, J. Zhao, and R. P. van Duyne, "Biosensing with plasmonic nanosensors," Nature Materials, vol. 7, no. 6, pp. 442-453, 2008.

[10] P. J. Jesuraj and K. Jeganathan, "Improved hole injection in organic light emitting devices by gold nanoparticles," RSC Advances, vol. 5, no. 1, pp. 684-689, 2015.

[11] F. Liu and J.-M. Nunzi, "Enhanced organic light emitting diode and solar cell performances using silver nano-clusters," Organic Electronics, vol. 13, no. 9, pp. 1623-1632, 2012. 
[12] C. Y. Cho, S. J. Lee, J. H. Song et al., "Enhanced optical output power of green light-emitting diodes by surface plasmon of gold nanoparticles," Applied Physics Letters, vol. 98, no. 5, article 051106, 2011.

[13] I. Lee, J. Y. Park, K. Hong, J. H. Son, S. Kim, and J.-L. Lee, “The effect of localized surface plasmon resonance on the emission color change in organic light emitting diodes," Nanoscale, vol. 8, no. 12, pp. 6463-6467, 2016.

[14] H. J. Kim, S. H. Lee, A. A. Upadhye et al., "Plasmon-enhanced photoelectrochemical water splitting with size-controllable gold nanodot arrays," ACS Nano, vol. 8, no. 10, pp. 1075610765, 2014

[15] Y. Xiong, R. Long, D. Liu et al., "Solar energy conversion with tunable plasmonic nanostructures for thermoelectric devices," Nanoscale, vol. 4, no. 15, pp. 4416-4420, 2012.

[16] S. Zou and G. C. Schatz, "Silver nanoparticle array structures that produce giant enhancements in electromagnetic fields," Chemical Physics Letters, vol. 403, no. 1-3, pp. 62-67, 2005.

[17] Y. Chu, E. Schonbrun, T. Yang, and K. B. Crozier, "Experimental observation of narrow surface plasmon resonances in gold nanoparticle arrays," Applied Physics Letters, vol. 93, no. 18 , article $181108,2008$.

[18] U. Guler and R. Turan, "Effect of particle properties and light polarization on the plasmonic resonances in metallic nanoparticles," Optics Express, vol. 18, no. 16, pp. 17322-17338, 2010.

[19] P. A. Mistark, S. Park, S. E. Yalcin et al., "Block-copolymerbased plasmonic nanostructures," ACS Nano, vol. 3, no. 12, pp. 3987-3992, 2009.

[20] Y. Lei and W.-K. Chim, "Shape and size control of regularly arrayed nanodots fabricated using ultrathin alumina masks," Chemistry of Materials, vol. 17, no. 3, pp. 580-585, 2005.

[21] M. Jung, W. A. el-Said, and J. W. Choi, "Fabrication of gold nanodot arrays on a transparent substrate as a nanobioplatform for label-free visualization of living cells," Nanotechnology, vol. 22, no. 23, article 235304, 2011.

[22] H. Masuda and M. Satoh, "Fabrication of gold nanodot array using anodic porous alumina as an evaporation mask," Japanese Journal of Applied Physics, vol. 35, Part 2, No. 1B, pp. L126-L129, 1996.

[23] T. Kondo, H. Masuda, and K. Nishio, "SERS in ordered array of geometrically controlled nanodots obtained using anodic porous alumina," Journal of Physical Chemistry C, vol. 117, no. 6, pp. 2531-2534, 2013.

[24] F. Matsumoto, M. Ishikawa, K. Nishio, and H. Masuda, "Optical properties of long-range- ordered, high-density gold nanodot arrays prepared using anodic porous alumina," Chemistry Letters, vol. 34, no. 4, pp. 508-509, 2005.

[25] I.-C. Chen, Y. H. Chen, Y. C. Wang, and M. H. Shih, "Plasmon resonance properties of silver-patterned glass substrates fabricated by anodic alumina oxide templates," Applied Physics A, vol. 112, no. 2, pp. 381-386, 2013.

[26] T. Kondo, F. Matsumoto, K. Nishio, and H. Masuda, "Surfaceenhanced Raman scattering on ordered gold nanodot arrays prepared from anodic porous alumina mask," Chemistry Letters, vol. 37, no. 4, pp. 466-467, 2008.

[27] Y. Wang, Y. Wang, H. Wang et al., "Hierarchical ultrathin alumina membrane for the fabrication of unique nanodot arrays," Nanotechnology, vol. 27, no. 2, article 025302, 2016.

[28] C. Y. Han, G. A. Willing, Z. Xiao, and H. H. Wang, "Control of the anodic aluminum oxide barrier layer opening process by wet chemical etching," Langmuir, vol. 23, no. 3, pp. 1564 $1568,2007$.

[29] H. Robatjazi, S. M. Bahauddin, L. H. Macfarlan, S. Fu, and I. Thomann, "Ultrathin AAO membrane as a generic template for sub-100 nm nanostructure fabrication," Chemistry of Materials, vol. 28, no. 13, pp. 4546-4553, 2016.

[30] G. Meng, T. Yanagida, K. Nagashima et al., "Facile and scalable patterning of sublithographic scale uniform nanowires by ultrathin AAO free-standing membrane," RSC Advances, vol. 2, no. 28, pp. 10618-10623, 2012.

[31] P. Prieto, V. Nistor, K. Nouneh, M. Oyama, M. Abd-Lefdil, and R. Díaz, "XPS study of silver, nickel and bimetallic silver-nickel nanoparticles prepared by seed-mediated growth," Applied Surface Science, vol. 258, no. 22, pp. 8807-8813, 2012.

[32] X. Wang, J. C. Yu, C. Ho, and A. C. Mak, "A robust threedimensional mesoporous ag/ $/ \mathrm{TiO}_{2}$ nanohybrid film," Chemical Communications, no. 17, pp. 2262-2264, 2005.

[33] Z. Jin, C. Liu, K. Qi, and X. Cui, "Photo-reduced $\mathrm{Cu} / \mathrm{CuO}$ nanoclusters on $\mathrm{TiO}_{2}$ nanotube arrays as highly efficient and reusable catalyst," Scientific Reports, vol. 7, no. 1, article 39695, 2017.

[34] I. Najdovski, P. R. Selvakannan, and A. P. O'Mullane, "Electrochemical formation of $\mathrm{Cu} / \mathrm{Ag}$ surfaces and their applicability as heterogeneous catalysts," RSC Advances, vol. 4, no. 14, pp. 7207-7215, 2014.

[35] W. Kautek and J. G. Gordon II, "XPS studies of anodic surface films on copper electrodes," Journal of the Electrochemical Society, vol. 137, no. 9, pp. 2672-2677, 1990.

[36] J. Hernandez, P. Wrschka, and G. S. Oehrlein, "Surface chemistry studies of copper chemical mechanical planarization," Journal of the Electrochemical Society, vol. 148, no. 7, pp. G389-G397, 2001.

[37] J. Radnik, C. Mohr, and P. Claus, "On the origin of binding energy shifts of core levels of supported gold nanoparticles and dependence of pretreatment and material synthesis," Physical Chemistry Chemical Physics, vol. 5, no. 1, pp. 172177, 2003.

[38] S. L. Westcott, J. B. Jackson, C. Radloff, and N. J. Halas, "Relative contributions to the plasmon line shape of metal nanoshells," Physical Review B, vol. 66, no. 15, article 155431, 2002.

[39] H. I. Muri and D. R. Hjelme, "LSPR coupling and distribution of interparticle distances between nanoparticles in hydrogel on optical fiber end face," Sensors, vol. 17, no. 12, article 2723, 2017.

[40] G. H. Chan, J. Zhao, E. M. Hicks, G. C. Schatz, and R. P. van Duyne, "Plasmonic properties of copper nanoparticles fabricated by nanosphere lithography," Nano Letters, vol. 7, no. 7, pp. 1947-1952, 2007.

[41] J. M. Luther, P. K. Jain, T. Ewers, and A. P. Alivisatos, "Localized surface plasmon resonances arising from free carriers in doped quantum dots," Nature Materials, vol. 10, no. 5, pp. 361-366, 2011.

[42] M. Kanehara, H. Koike, T. Yoshinaga, and T. Teranishi, "Indium tin oxide nanoparticles with compositionally tunable surface plasmon resonance frequencies in the near-IR region," Journal of the American Chemical Society, vol. 131, no. 49, pp. 17736-17737, 2009. 


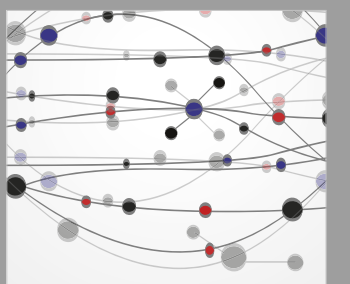

The Scientific World Journal
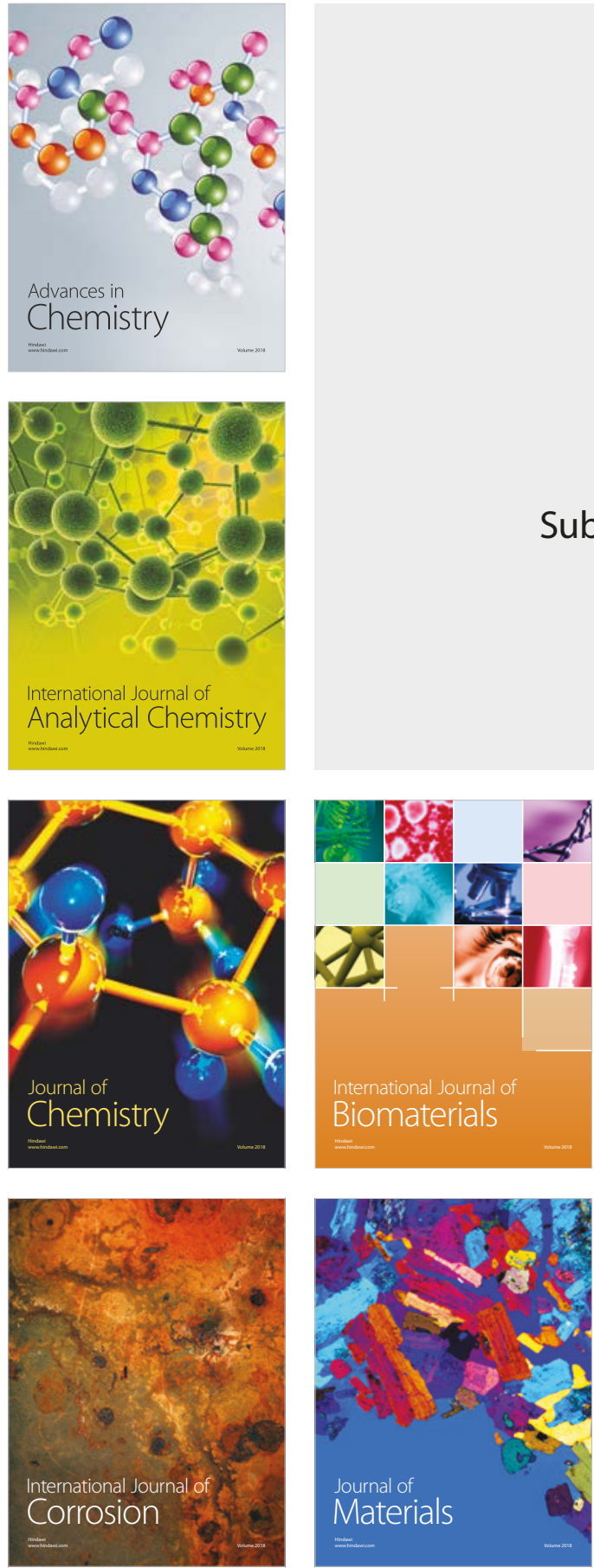

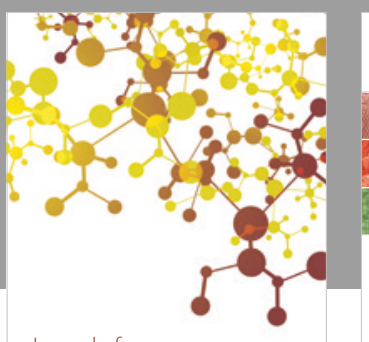

Journal of

Applied Chemistry
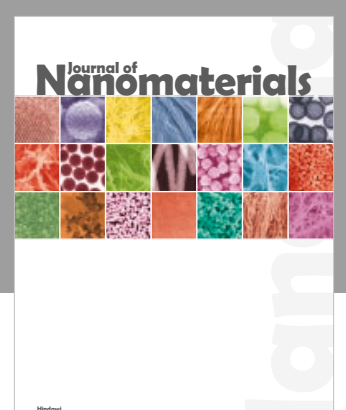

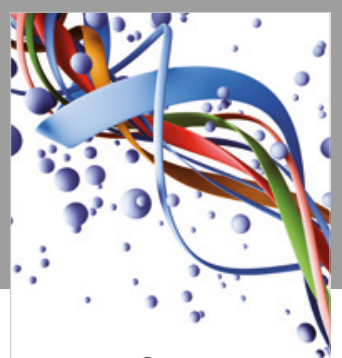

Scientifica

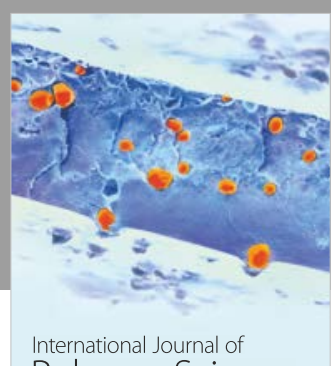

Polymer Science

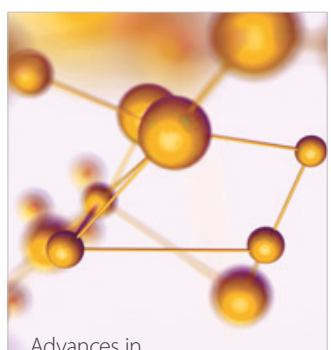

Physical Chemistry
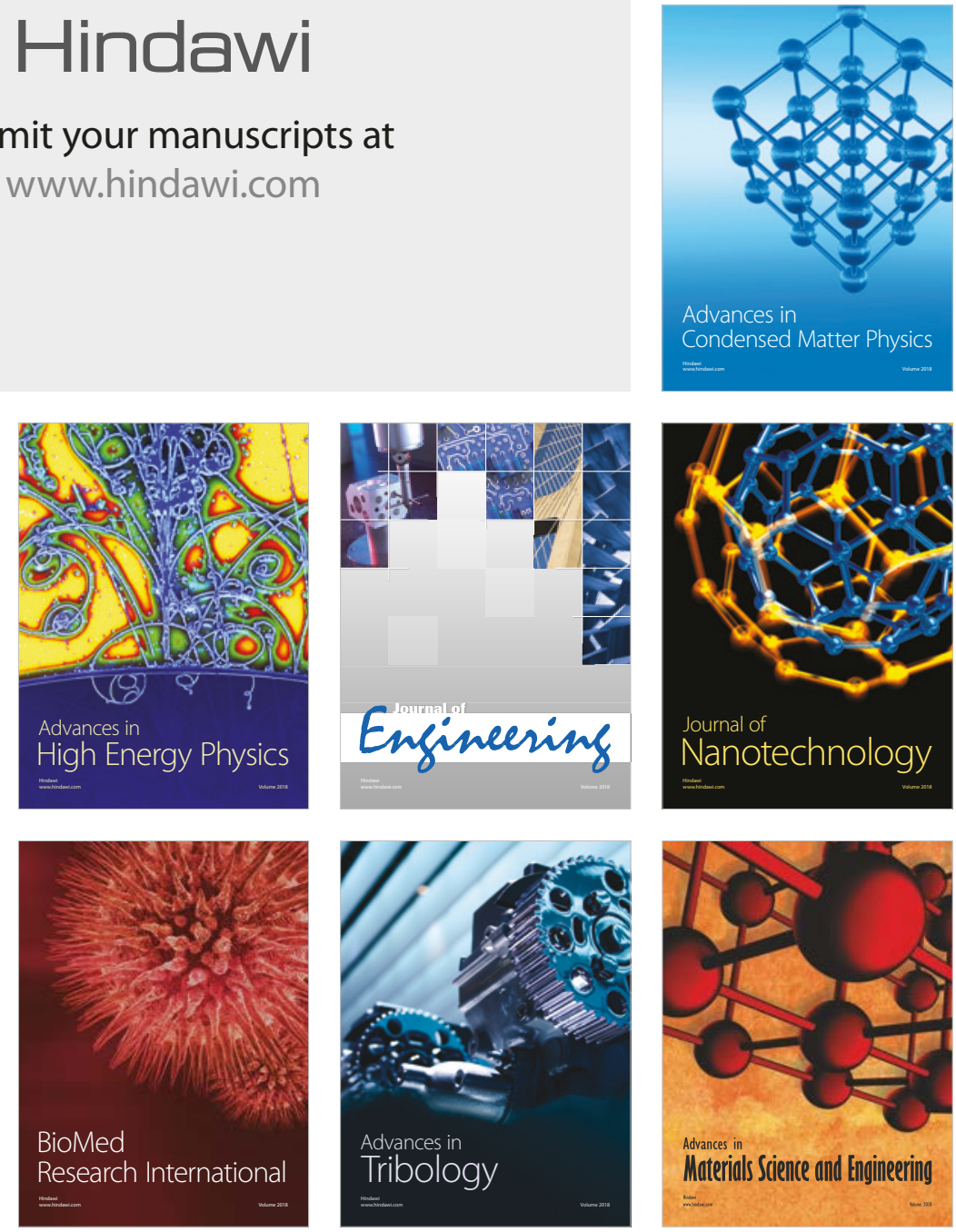